\title{
A Case of Femoral Arterial Bleeding by an Unknown Origin Metastatic Groin Lymph Nodes Carcinoma
}

\author{
Eiji Nakamura, MD, Takeshi Oda, MD, Hiroshi Yasunaga, MD, and Shigeaki Aoyagi, MD
}

We report a case of femoral arterial bleeding complicated with hemorrhagic shock caused by bacterial infection attributed to an inguinal lymph node metastasis of carcinoma of unknown primary. Because of severe preoperative condition, a venous patch plasty of ruptured artery, and omentopexy for the groin was performed as a less invasive surgery. But the recurrence of bleeding was occurred postoperatively.

A staged operation, hemostasis with a venous patch plasty at a first stage, and an extra-anatomical bypass soon after improvement of shock condition in a second stage, can be one of surgical procedures to save the lives and salvage limbs.

Keywords: femoral arterial bleeding, groin lymph nodes, groin infection

\section{Introduction}

Carcinoma of unknown primary (CUP) is defined as the presence of histologically proven metastatic cancer for which the site of origin cannot be identified at the time of diagnosis despite comprehensive diagnostic procedures, and it is one of the ten most frequent cancers and is the fourth most common cause of cancer-related death. ${ }^{1)}$ Generally, $10 \%$ to $40 \%$ of CUP patients are diagnosed with metastasis in the lymph nodes, while the remaining patients present with metastasis in their internal organs. ${ }^{2)}$ Metastasis to the lymph nodes is most frequent in the head and neck but that in the inguinal region and lower limb is relatively rare. ${ }^{2)}$ However, a metastatic inguinal lymph node carcinoma frequently involves the femoral vessels in its advanced stage and leads to massive bleeding and even death. ${ }^{3,4)}$

Department of Cardiovascular Surgery, St. Mary's Hospital, Kurume, Fukuoka, Japan

Received: October 12, 2015; Accepted: December 14, 2015

Corresponding author: Eiji Nakamura, MD. Department of Cardiovascular Surgery, St. Mary's Hospital, 422 Tsubukuhonmachi, Kurume, Fukuoka 830-8543, Japan

Tel: +81-942-35-3322 (ext 6182), Fax: +81-942-34-3115

E-mail: e-nakamura@st-mary-med.or.jp
In this paper, we report a case of femoral arterial bleeding caused by bacterial infection attributed to an inguinal lymph node metastasis of a CUP in an elderly patient.

\section{Case Report}

A 79-year-old man was emergently transferred to our hospital for treatment of pulsatile massive bleeding from the eroded ulceration in the left groin with hemorrhagic shock. He noticed swelling of the lymph nodes in the left groin 15 months previously, and then the patient was diagnosed with metastatic carcinoma of the lymph nodes, which was pathologically classified as squamous cell carcinoma (SCC), after biopsy of the affected lymph node. Despite of close examinations using extensive diagnostic procedures, which included measurement of tumor markers, ultrasonography, computed tomography (CT), magnetic resonance imaging, gastrointestinal endoscopy, and combined ${ }^{18} \mathrm{~F}$-fluorinefluorodeoxyglucose positron emission tomography and computed tomography (FDG-PET/CT), the primary origin was not identified. FDG-PET showed high levels of FDG uptake in the left groin but significant FDG uptake was not detected in any other site such as the abdomen, the genitals and the lower extremities (Fig. 1). Based on these findings, a diagnosis of CUP with inguinal lymph node metastasis was made, and radical inguinal lymphadenectomy was judged to be difficult. Then, the patient underwent radiation therapy, but it was not effective to suppress expansion of the metastatic lymph node carcinoma. During the following 7 months, the expanded metastatic lymph node carcinoma eroded the skin with occurrence of ulceration, infection, and femoral vessel involvement. A small amount of bleeding from the ulceration repeatedly occurred during the last 4 months. CT performed one month previously revealed the large, irregular and poorly bordered mass extended from left pelvic region to the left groin with necrosis of its central part with involvement of the iliac and femoral arteries and a fistula to the skin (Fig. 2A). Thrombosis of the left femoral and iliac veins and cancerous invasion to the pelvis were also demonstrated on CT (Figs. 2B, 2C). On admission, the patient was in circulatory collapse 


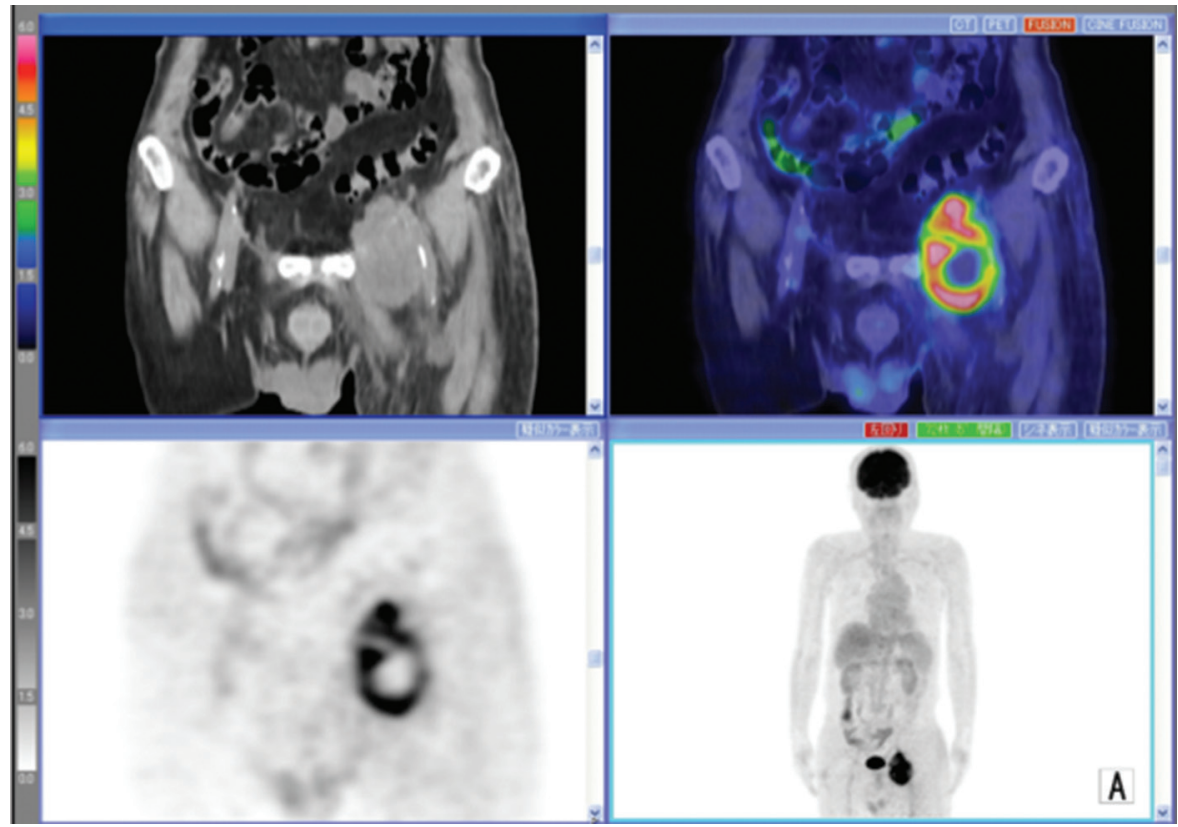

Fig. 1 FDG-PET/CT showed high levels of FDG uptake only in the left groin. FDG-PET/CT: ${ }^{18} \mathrm{~F}$-fluorine-fluorodeoxyglucose positron emission tomography/computed tomography

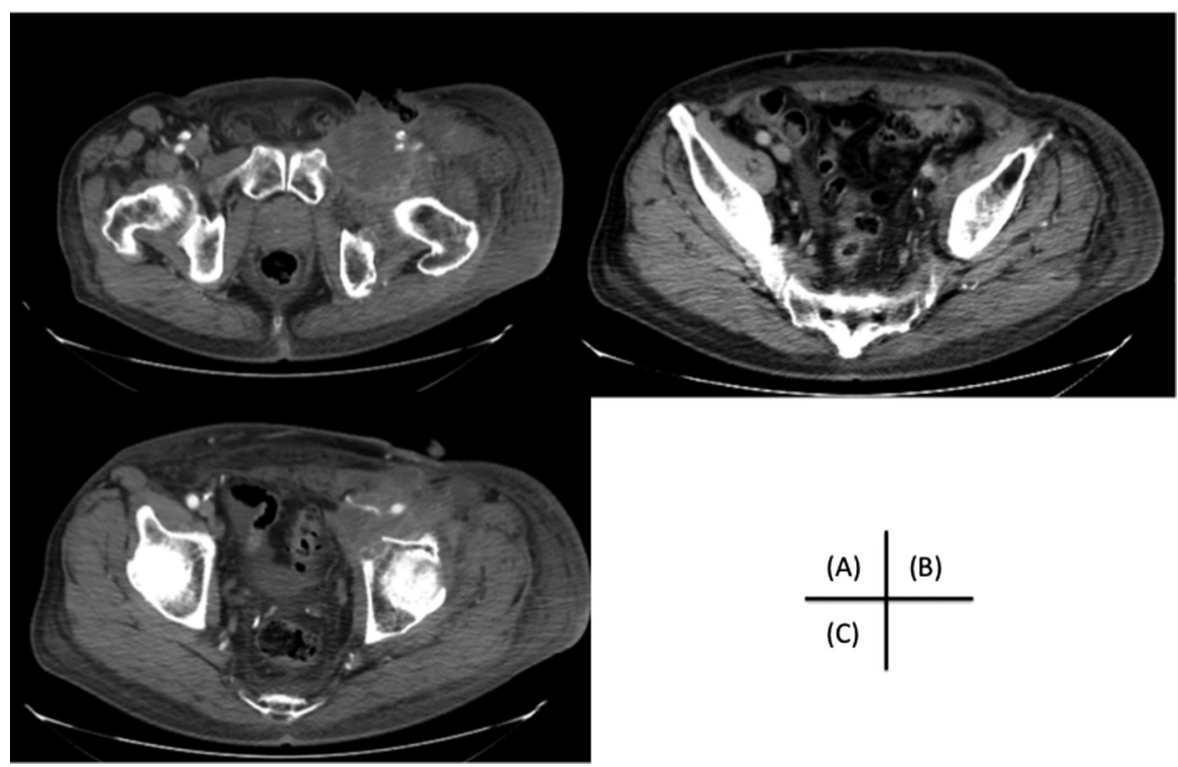

Fig. 2 CT showed necrotic tumor around the femoral arteries at the left groin and skin erosion (A), thrombosis of the left external iliac vein (B), and cancerous invasion to the pelvis (C). CT: computed tomography

with a blood pressure of $72 / 38 \mathrm{mmHg}$ and an irregular heart rate of $116 / \mathrm{min}$. The eroded ulceration of the left groin was $5 \times 5 \mathrm{~cm}$ in size and emitted a foul odor resulting from infection and necrosis. The pulsatile bleeding caused by rupture of the left common femoral artery was macroscopically confirmed. The pulsation of the posterior tibial and pedal dorsal arteries was not palpable and Doppler signals over the arteries were not audible because of severe leg edema and low blood pressure. No abnormality was found in the penis and the anorectum, and cervical, supraclavicular and axillary lymph nodes were not palpable. Hematologic examination showed red blood cell count of $138 \times 10^{4} / \mathrm{mm}^{3}$, hemoglobin of $4.0 \mathrm{~g} / \mathrm{dl}$, white blood cell count of $16810 / \mathrm{mm}^{3}$ and C-reactive protein (CRP) of 13.8 $\mathrm{mg} / \mathrm{dl}$. Because of his preoperative critical physical condition and poor prognosis due to extensive lymph node metastases, minimal invasive management, hemostasis and limb salvage, was planned. 


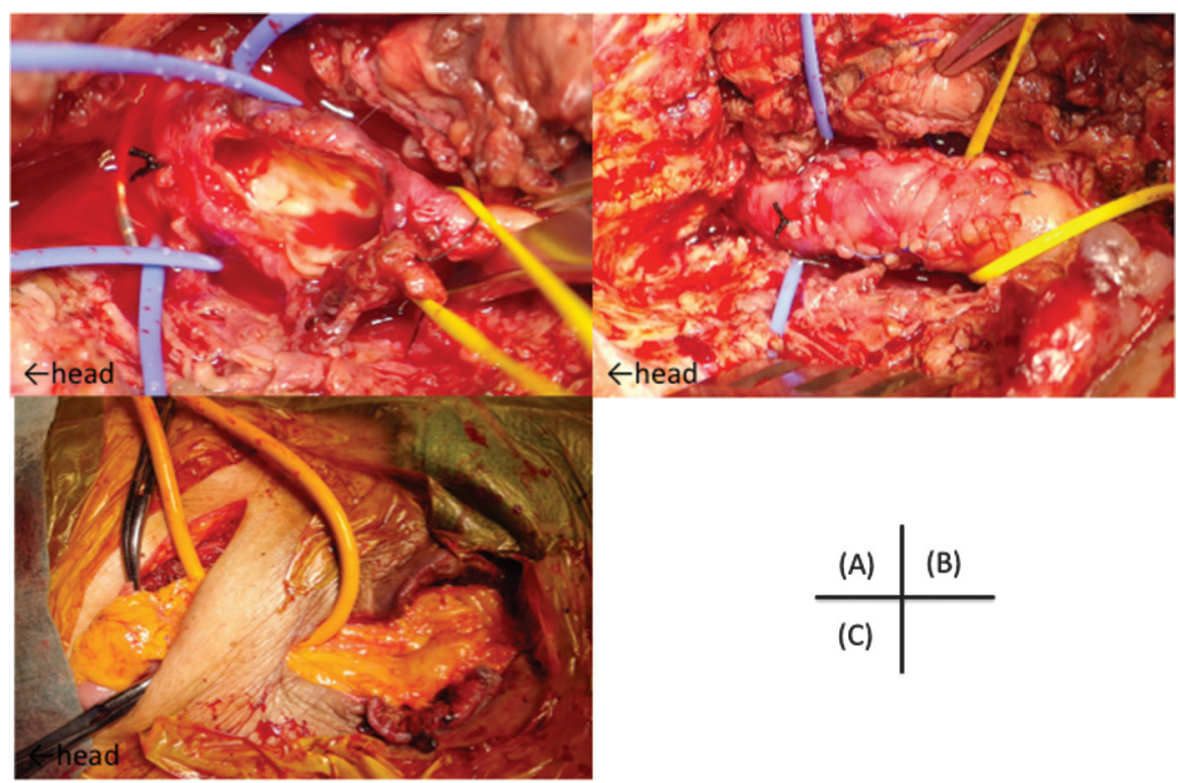

Fig. 3 Intraoperative photograph showed the ruptured common femoral artery with $15 \mathrm{~mm}$ hole at the long axis surrounded by melted adventitia (A), venous patch plasty with autologous saphenous vein for ruptured common femoral artery $(\mathbf{B})$, and the omentopexy for left groin (C).

At surgery, proximal control was obtained through a retroperitoneal approach to the external iliac artery. The left femoral artery was totally involved in the necrotic and infected tissue of the mass, and the rupture of the left common femoral artery, a size of $15 \mathrm{~mm}$, caused by bacterial infection was found (Fig. 3A), and patch repair of the ruptured femoral artery with an autologous saphenous vein was performed after debridement of the arterial wall around the rupture site (Fig. 3B). In addition to limited debridement of the necrotic tissue and irrigation of the lesion using saline solution, coverage with an omental flap was used to preclude extension of the infection to the repaired artery (Fig. 3C). Culture of the debrided arterial wall was positive for Pseudomonas aeruginosa but histological examination of the arterial wall was not performed.

Postoperatively, the patient recovered from shock condition soon after surgery without clinical symptoms and signs of pulmonary thromboembolism such as severe hypoxemia and hypocapnemia of arterial blood (partial pressure of oxygen (PO2) $68.9 \mathrm{mmHg}$, partial pressure of carbon dioxide (PCO2) $32.1 \mathrm{mmHg}$ while breathing room air, respiratory rate $17 / \mathrm{min}$ ). A serum CRP level was quickly decreased from $19.2 \mathrm{mg} / \mathrm{dl}$ on postoperative day 3 to $6.3 \mathrm{mg} / \mathrm{dl}$ on postoperative day 10 by switch of sulbac$\operatorname{tam}(2 \mathrm{~g} /$ day $)$ to carbapenem ( $1.5 \mathrm{~g} /$ day $)$ according to the results of a sensitivity test for Pseudomonas aeruginosa. A serum procalcitonin level was also decreased from 11.1 $\mathrm{ng} / \mathrm{ml}$, preoperatively, to $1.4 \mathrm{ng} / \mathrm{ml}$ on postoperative day 7 . However, expansion of the eroded ulceration of the left groin continued. At that time, despite our strong proposal for the second operation, extra-anatomical bypass grafting and resection of the affected femoral artery, the patient obstinately denied further invasive treatment because of his poor prognosis. Finally, he died of recurrent massive bleeding 14 days after the operation. Autopsy was not permitted.

\section{Discussion}

CUP is a histologically confirmed cancer which is manifested in advanced stage, with no identifiable primary origin after the use of extensive diagnostic procedures. According to studies in Europe, ${ }^{2,5,6)}$ CUP accounts for 3\% to $5 \%$ of all cancers, although the reported incidence of CUP extends to a wide range because of the difficulty in defining CUP. A study analyzing 26641 CUP patients demonstrated that 9633 patients $(36.2 \%)$, in total, were diagnosed with lymph node metastasis. Among various locations of the lymph node, the inguinal lymph node was affected in $182(1.9 \%)$ of the 9633 CUP patients. ${ }^{2)}$

To identify a primary tumor, an extensive diagnostic work-up is required because treatment of CUP is guided by the suspected origin of the primary tumor. In addition to some imaging techniques such as ultrasonography, CT and magnetic resonance imaging, the employment of FDG-PET or combined FDG-PET/CT, which allows whole-body tumor detection, is recommended for detailed diagnosis of CUP, and they have proven to be useful in patients with CUP for the detection of the primary tumor. ${ }^{1,7}$ Seve and 
colleagues $^{7}$ indicated that FDG-PET detected the primary lesion in 91 of 221 patients who had metastases from a CUP, yielding a detection rate of $41 \%$. In our patient, we also used FDG-PET/CT to investigate the primary origin, however, FDG-PET demonstrated high levels of FDG uptake only in the left groin, and significant FDG uptake was not detected in any other site of the body (Fig. 1).

Metastatic SCC of the inguinal lymph node has been frequently reported in penile cancer. ${ }^{3,4}$ In our patient, therefore, lymph node metastasis of penile cancer was most suspected, however, a definite abnormality was not identifiable even by FDG-PET/CT in the penis.

Generally, with expansion of the tumor, metastatic SCC of the inguinal lymph node evolves to central necrosis, skin erosion with infection and femoral vessel involvement, causing massive bleeding and even death. Successful treatment of arterial bleeding and infections in the groin requires excision of the affected vascular segment, adequate debridement, and restoration of blood flow to the limb by either in situ or extra-anatomical bypass. ${ }^{8}$ Engin and colleagues ${ }^{8)}$ have concluded that vascular infections of the groin can be cured by application of one of revascularization techniques including lateral femoral bypass, obturator bypass, femorofemoral bypass, or in situ reconstruction with a homograft. However, in our patient, preoperative physical condition and poor prognosis due to the extensive lymph node metastasis were considered to be too serious to have a large operation, which requires long time, and less invasive surgery, repair of the femoral artery with an autologous venous patch, was selected to save his life and limb. Arora and colleagues ${ }^{9)}$ have emphasized that common femoral artery ligation and local debridement are safe treatment modalities for infected femoral artery pseudoaneurysms, if there is an intraoperative Doppler signal over a pedal artery during test occlusion of the distal external iliac artery or common femoral artery. Ligation of the common femoral artery is also one of simple and less invasive procedures, however, as considering patient's age and the absence of the peripheral Doppler signals, ligation of the common femoral artery was considered to be risky for limb salvage in our patient.

Retrospectively, in this patient, as the second operation, a supplementing extra-anatomical bypass grafting and resection of affected femoral artery with closure of the stumps should have been performed soon after recovery from shock condition, at the latest, within 7-10 days after the first operation, and a lateral femoral bypass or axillo-popliteal bypass grafting ${ }^{10)}$ could have been an appropriate procedure in this patient because extension of metastatic tumor to the obturator foramen was found on CT and during the operation.

In conclusion, we report a case of femoral arterial bleeding complicated with hemorrhagic shock caused by bacterial infection attributed to inguinal lymph node metastasis of CUP. In patients with hemorrhagic shock due to infection of the femoral vessels, a staged operation, hemostasis of arterial bleeding and coverage of the repaired artery by a viable tissue at a first stage, and then an extra-anatomical bypass and resection of the infected segment of the femoral artery with closure of the femoral stumps soon after improvement of shock condition in a second stage, can be one of surgical procedures to save their lives and salvage limbs.

\section{Disclosure Statement}

All authors have no conflict of interest.

\section{Author Contributions}

Study conception: EN

Writing: EN

Critical review and revision: all authors

Final approval of the article: all authors

Accountability for all aspects of the work: all authors

\section{References}

1) Kwee TC, Kwee RM. Combined FDG-PET/CT for the detection of unknown primary tumors: systematic review and meta-analysis. Eur Radiol 2009; 19: 731-44.

2) Hemminki K, Bevier M, Hemminki A, et al. Survival in cancer of unknown primary site: population-based analysis by site and histology. Ann Oncol 2012; 23: 1854-63.

3) Agostinucci A, Sandrone N, Baracco C, et al. Extraanatomic lateral bypass combined with embolization of the femoral stump for management of groin infection and bleeding due to metastatic carcinoma of the penis. Intract Cardiovasc Thorac Surg 2011; 12: 860-2.

4) Ferreira U, Reis LO, Ikari LY, et al. Extra-anatomical transobturator bypass graft for femoral artery involvement by metastatic carcinoma of the penis: report of five patients. World J Urol 2008; 26: 487-91.

5) van de Wouw AJ, Janssen-Heijnen ML, Coebergh JW, et al. Epidemiology of unknown primary tumors: incidence and population-based survival of 1285 patients in Southern Netherlands, 1984-1992. Eur J Cancer 2002; 38: 409-13.

6) Randén M, Rutqvist LE, Johansson H. Cancer patients without a known primary: incidence and survival trends in Sweden 1960-2007. Acta Oncol 2009; 48: 915-20.

7) Sève P, Billotey C, Broussolle C, et al. The role of 2-deoxy2-[F-18]fluoro-D-glucose positron emission tomography in disseminated carcinoma of unknown primary site. Cancer 2007; 109: 292-9.

8) Engin C, Posacioglu H, Ayik F, et al. Management of vascular infection in the groin. Tex Heart Inst J 2005; 32: 529-34.

9) Arora S, Weber MA, Fox CJ, et al. Common femoral artery ligation and local debridement: a safe treatment for infected femoral artery pseudoaneurysms. J Vasc Surg 2001; 33: 990-3.

10) Keller MP, Hoch JR, Harding AD, et al. Axillopopliteal bypass for limb salvage. J Vasc Surg 1992; 15: 817-22. 